\title{
Vision Based Position Control for Vertical Take-off and Landing (VTOL) Using One Singular Landmark
}

\author{
Hairol Nizam Mohd Shah ${ }^{1}$, Zalina Kamis ${ }^{1}$, Azhar Ahmad ${ }^{1}$, Mohd Rizuan Baharon², Muhd Akmal Noor Rajikon ${ }^{3}$ \\ \& Kang Hui Hwa ${ }^{1}$ \\ ${ }^{1}$ Center for Robotics and Industrial Automation, Faculty of Electrical Engineering, Universiti Teknikal Malaysia \\ Melaka, Malaysia \\ ${ }^{2}$ Department of Computer System and Communication, Faculty of Communication and Information Technology, \\ Universiti Teknikal Malaysia Melaka, Malaysia \\ ${ }^{3}$ Language Center and Human Development, Universiti Teknikal Malaysia Melaka, Malaysia \\ Correspondence: Hairol Nizam Mohd Shah, Center for Robotics and Industrial Automation, Faculty of Electrical \\ Engineering, Universiti Teknikal Malaysia Melaka, Malaysia. E-mail: hnizam@utem.edu.my
}

The research is financed by (Sponsoring information)

Received: May 24, 2019

doi:10.5539/mas.v13n9p33
Accepted: August 16, 2019

Online Published: August 22, 2019

\begin{abstract}
This project presents a vision based position control for Vertical Take-off and Landing (VTOL) to recognise a singular landmark for landing and take-off. Position control can provide safe flight and an accurate navigation. The circle landmark which used is an artificial landmark at known locations in an environment. Initially, a camera mounted on VTOL facing downward detecting landmarks in environments. A single circle used as landmark and VTOL will be control the position to reach the landmark. The images from the down-looking camera provided vision data to estimates position of VTOL from landmark. A mathematical method based on projective geometry using to locate VTOL on desired landmark from projected point in capture image. By compute the $\mathrm{x}-\mathrm{y}$ coordinates of the VTOL with respect to landmark, height of camera above landmark will be obtained. VTOL can localize itself in known environment with pose estimation from landmark. The graphic user interface system (GUI) generate by MATLAB software is used to communicate with VTOL to control the VTOL position
\end{abstract}

Keywords: Vertical Take-off and Landing (VTOL), projective geometry, graphic-user-interface system (GUI), recognition algorithms

\section{Introduction}

A vertical-takeoff-and-landing (VTOL) is defined as aircraft that can hover, take-off and land vertically without using runway, for example, helicopter. Unlike conventional (non-VTOL) airplanes, there are few of peoples have been inside a VTOL since it is mainly used for some critical tasks such as search and rescue, accident victims. The VTOL is contributed in the the survival operation by simply reduce reaction time of getting medical opportunities and critical situations is reduced.

The traditional recognition techniques based on geometric models is replaced by vision-based visual techniques. Vision based method is simpler compares to traditional method. Nowadays, as many new technology developed on image processing, circular shape recognition and position estimation using computer vision or image processing is one of significant research subject in application fields such as mobile robots, intelligent Closed-circuit television (CCTV), and target recognition. The frame of captured image is extracted the landmark feature, then thresholding, filtering and obtain the circular object parameter in the frame. From the obtained circle parameter, a position estimation mathematical equation is developed by studying the relationship between area size of circle and the actual height of VTOL.

VTOL is difficult to obtaine accurate geometric model to estimate current position using Global Positioning System (GPS) coordinate system. GPS receiver is the most famous sensor used in navigation task for VTOL including search and rescue, wild fire suppression, industrial and agricultural application. However, GPS is difficult to use since GPS satellite signal too weak, it can not receive data in indoor environment. Besides 
that,GPS accuracy affected in urban area due to some satellite signals blocked by buildings that will resulting positioning errors. Therefore, the solution of localization problem is developed vision-based navigation systems use wireless pinhole camare to recognize a desired landmark for positioning task in urban area or even indoor environments.

In some environment that is complex, hard to find out landmark since too much object. Hence, this project using a circular landmark only. The VTOL above artificial circular landmark estimate distance between landmark and VTOL. A landmark images at a certain position is capture, then the distance of circular objects from the camera can be analyze with image circular parameter obtained from the frame. A mathematical models is develop by from the relationship of area size of circle and the actual position between camera of VTOL and ground floor. In this way, the resulting system is able to recognise artificial circular landmark, estimate position of VTOL on the landing area for search and rescue mission in disaster, for example, earthquake. By using wireless pinhole camera, VTOL able to identify the location of safety area, then VTOL locate survivors in the safe landing area with desired artificial landmark. Therefore, this project will focus on implement of vision-based landmark recognition, tracking and position estimation algorithm for VTOL using wireless camera system.

\section{Method}

The study of VTOL mainly contribute in positioning task. Position of target estimated by a mobile robots and used for localization (D. Eberli et al., 2010; Gohring et al., 2006). D. Eberli et al. (2010) have proposed a vision based algorithm for pose estimation and set-point control using a camera mounted on-board a VTOL. Camera pose estimation is based on features landmark included camera calibration (D. Eberli et al., 2010; Gohring et al., 2006). In the process of landmark recognition, circle which used as landmark showed elliptic appearance in perspective projection for estimate VTOL position. VTOL is controlled through image-based algorithm to landing on respective target position (D. Eberli et al., 2010; Gohring et al., 2006).

The vision based position control of VTOL is equivalent to the external camera calibration problem. There are two method to solve this problem. First method is using stereo camera system with two camera place on both left and right side of VTOL. The pose of the camera obtained from the sequence of overlapping images which proposed by GAO ( $\mathrm{Lu}$ fang Gao et al., 2007). A vision-based algorithm used to extract particular two-dimensional (2D) horizontal environmental image features and vertical edges. This method (Gohring et al., 2006) is commonly used in recognizing more than one landmark in a complex environment. Since, single circular landmark instead of multi-landmark using in this project, single camera system is more suitable to recognizing landmark.

Second method is using a mono camera system and desired landmarks to obtained position of camera. In Ray N et al. (2005) and Myong et al. (2003), the researchers used the relation of detected target images in perspective to other object in the same camera image for target pose estimates in a global coordinate system. The spatial relation of objects with respect to landmarks by using single camera system gives some advantages such as errors in feature detection are correlated, independent robot localization information, asynchronous communication and high efficient communicating method (Gohring et al., 2006).

In order to locate mobile robot towards target position, some solution is proposed by researcher from world. G. Jang et al. (2003) proposed using artificial and natural landmarks by computing the local Zernike moments to solved the localization for the mobile robot. Haibo et al. (2005) conducted an experiment finding parallel lines and vertical lines using the Hough transform. Two lines is intersected and form a cross point. In this experiment, the distance from vehicle to the cross point is calculated as cross point used as target. An algorithm is used to mapping environment to the feature points (Lu fang Goa et al., 2007).

The one of most common way to estimate position of VTOL is triangular method.The position of landmarks is known in a world-centered coordinate system (WCCS). In order to calculate landmark coordinates in a camera mounted on board VTOL to localize its position by calculating the camera's viewpoint through looking at the landmarks (Myong et al., 2003; G. Jang et al., 2003).

Pifu et al. (2004) proposed by using minimum three feature points and image captured by mono camera to solve camera position and orientation approach. Lu Wang et al. (2007) examined alternative iterative approaches with at least two line and point correspondences to same problem. Both of them realize that feature points 3D coordinates in a camera centered coordinate system (CCCS) need computed to obtain a closed-form solution through the geometric transformations to map the 3D points from CCCS to WCCS. In this project, triangulation method is using for updating position VTOL from time to time after capture landmark image matches with reference landmark image at a certain height, for example, $55 \mathrm{~cm}$ above ground. 
Tracking landmark using shape matching is presented by Qi et al. (2006). Before doing shape matching, first step is searching landmark by from background environment. After that, apply some image preprocessing techniques such as segmented, determine edge onto captured landmark images. Second step is extracted landmark shape from previous frame, shape matching, and generate updating landmark image in next frame until find out precise position of artificial circular landmark.

The recognition of circular shapes is a common task in computer vision and image recognition. Recognition of circular shape landmark has been researched using a variety of techniques. Circular shape recognition can be done through image processing, a gray-scale image convert to binary using edge detection techniques and parameter of circular is compute to build a position estimation mathematical models. There are few methods to detecting the shape of circle. The first method is by circle fitting method. Algorithm of moment invariants and Euclidean distances has been used for computation that can be adapted to measure the parameter of circular. Set of points using the least-squares method to fitting circles or having a iterative approach to obtain circular equation (Arvacheh et al., 2006). This method build a more accurate mathematical equation is suitable for position control used in this project.

The second method is active contour (snakuscules) grouping algorithms. By using this technique, a continuous boundary of circular object is detected without calculating the gradients of boundaries. This method reshape an initial curve provided by segmentation technique, an object is extract from a given image based on thresholding and morphological operations. In an iterative process it is dynamic moved according to an appropriately designed energy function until it finds the local optimum. The energy function calculates the difference between object features and background features. An iterative algorithm has been developed in order to determine the object boundary. This method also be employed for tracking moving objects (Thevenaz et al., 2006; Arvacheh et al., 2006; Ray et al., 2005).

Figure 1 show a microscopy image of a plant cell with ten times magnification. A variety of cell shape and sizes is seen show in Figure 2. The initial configuration of snakuscules cover in 640 x 360 image size. In the Figure 2.3, all cell found their optimal boundary. From the Figure 3, it can observe that are some bright circular blobs obtained after apply snakuscules. By using snakuscules method, the size of filter can be vary and analytically find out the gradient of the circular in respect to defining parameter of circle.

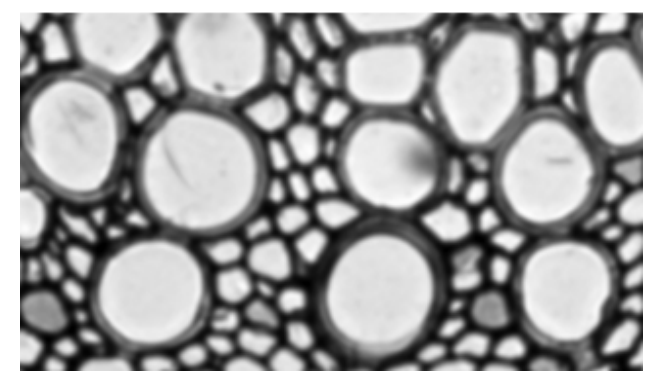

Figure 1. A plant cell under 10 times magnification

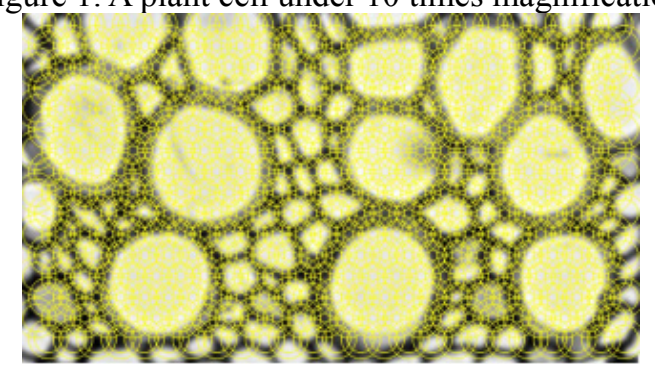

Figure 2. Initial configuration of snakuscules

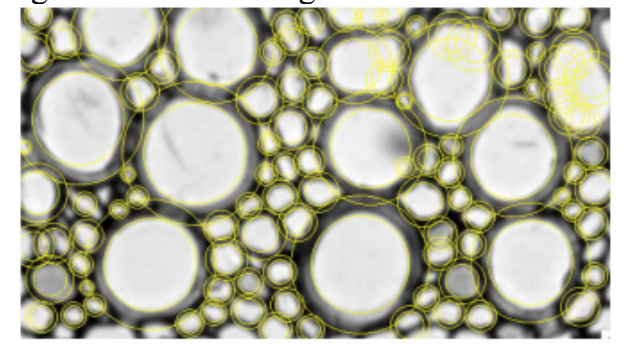

Figure 3. Final configuration of snakuscules show a sharp image. 
The third method used for detecting circular shape is he Circle Hough Transform (CHT). By using this method, the circle parameter such as a center point, $(x, y)$ and a radius, $r$ is determined. HT algorithms used to detect circles computationally more complex than circle fitting algorithms as a large number of parameters involved in describing the shapes. To determine a circle, it is three-dimensional parameter space $(x, y, r)$ is necessary to find out.

\section{Methodology}

\subsection{Projects Methodology Flowchart}

The methodology for this research consists of 3 major stages. The stages are landmark recognition, determine parameter of circular shape and pose estimation of VTOL. The landmark recognition stage includes the image acquisition and preprocessing. Landmark recognition based on the most significant part of input image. First, a set of image frames that include artificial landmark then a circular shape object with a diameter of $6 \mathrm{~cm}$ is captured from different viewpoints and orientation. The objects were placed relatively close to the camera.

A circular shape detection algorithm is used to find out the circular object in captured image from the running GUI. The image then converts in gray scale to identify whether the object is circular shape by circular shape recognition algorithm. The irrelevant image features are eliminates and thus reduce image computation.

After the object is identified by circular shape detection algorithm, its parameter of the circle included centroid and the area size of the circle in pixel should be obtained to estimate the VTOL height above circular landmark. The pose estimation performed by using mathematical models was develops based on the relationship between area size of circle and the actual height of VTOL. The third step is computing the approximate position of VTOL based on the parameter of object. By applying mathematical equation, the position of VTOL is estimated from in recognized landmark. Figure 4 show a flow chart for research methodology proceses.

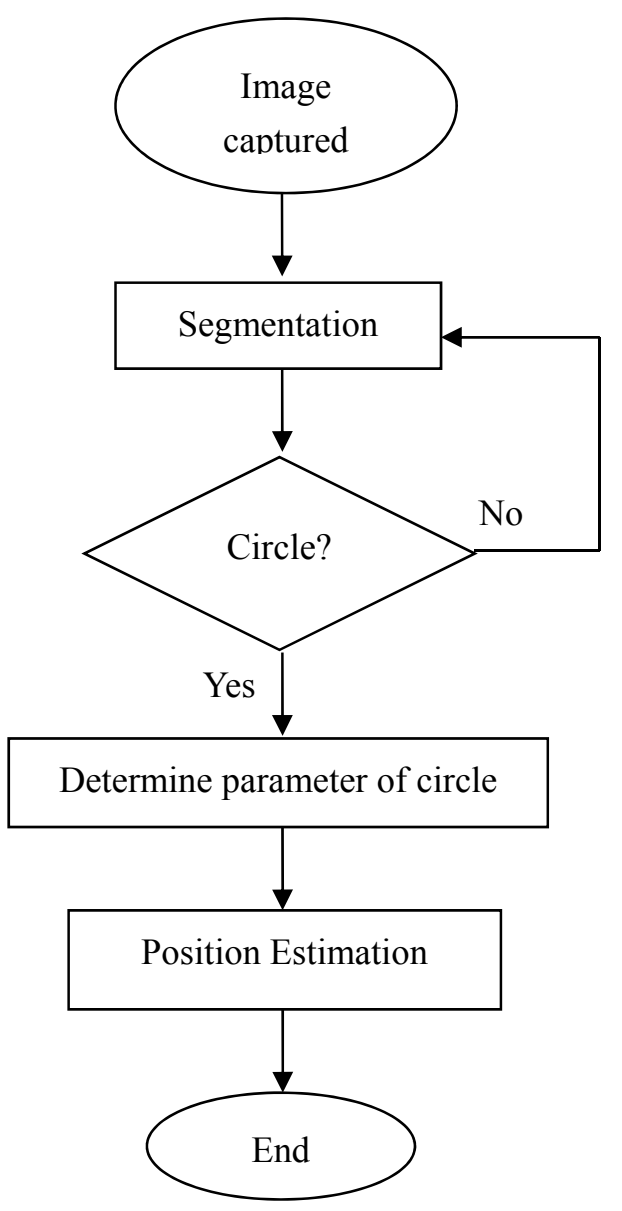

Figure 4. Flow chart research processes 


\subsection{Developed Circular Shape Detection Algorithm}

The concept of feature recognition aims at computing extractions of image information and making robust decisions at every image point whether there is an frame feature such as circular, rectangular or triangle shape at that point. The recognition of circular object is an initial process for the tracking using two methods for clear cut object detection. It is required due to several reasons which are detected object in one frame and separately detected object in another frame can obtain mean of another object.

\subsection{Interfacing the Camera with the Program}

The hardware interfacing is consisting of wireless camera, RG 59 video cable and GUI interface. The wireless camera has been mounted on the VTOL during tracking the circular shape object when landing and flying. For a vision ranging system, accuracy of recovered distance depends on the imaging resolution. An accurate system results in precise pose estimation. The distance that between the camera mounted on VTOL with respect to the landmark object is important to select its ranging distance. Figure 5 and 6 show the interfacing hardware will be used in this research. The VTOL testing flight during landing and take off are show in Figure 7 and 8.

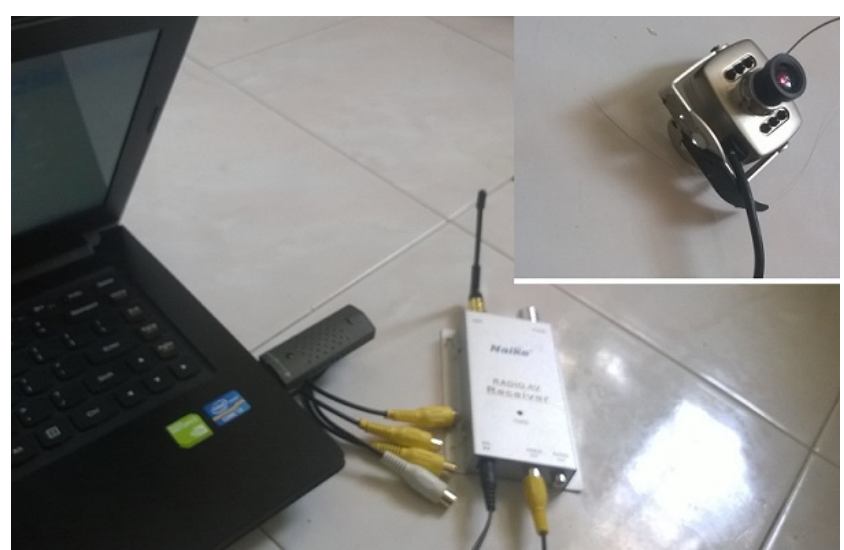

Figure 5. Wireless camera and receiver

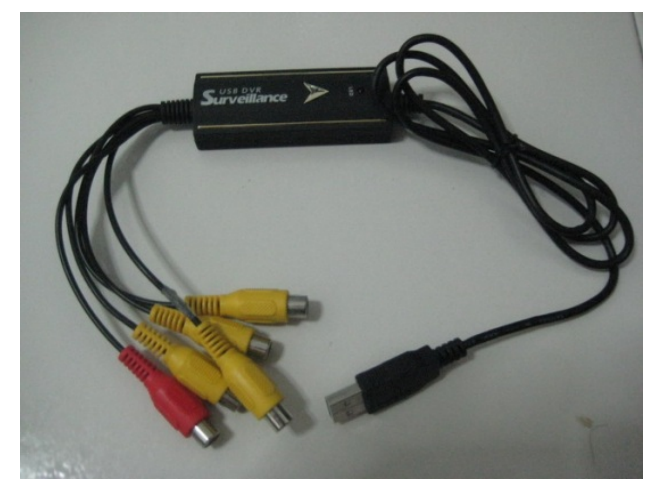

Figure 6. RG59-to-USB converter

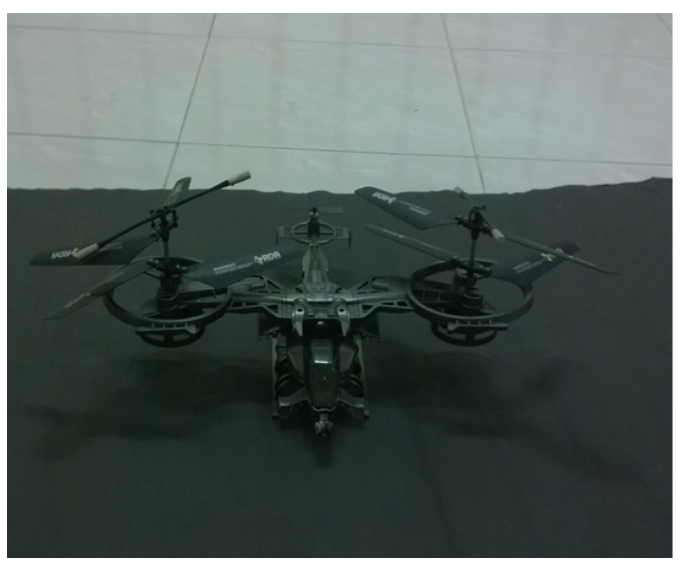

Figure 7. VTOL landing 


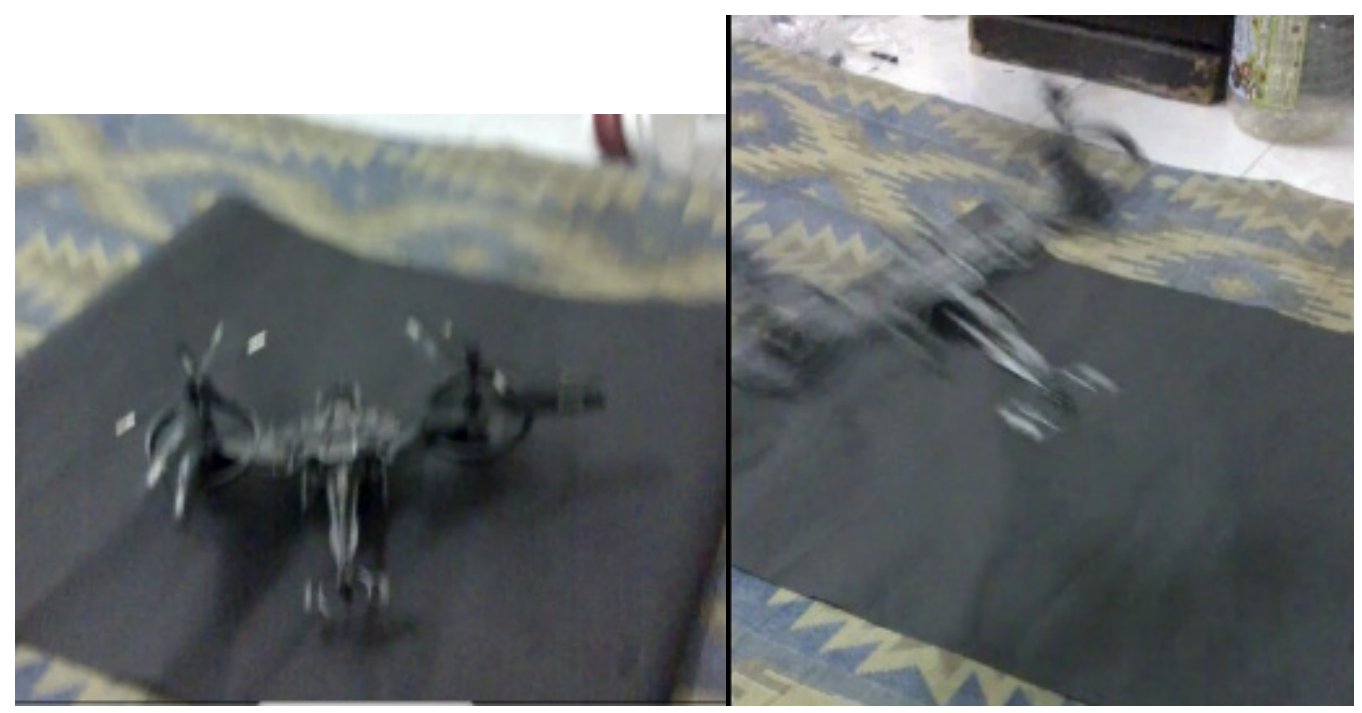

Figure 8. VTOL take off

\subsection{Image Processing Method}

Image preprocessing is required to enhance image features also to elimated the noise of the image. The preprocessing of the image able to enhance image quality and improve the recognition of circular shape objects. A circular shape detection algorithm on the image will obtain centroids coordinates $(\mathrm{x}, \mathrm{y})$ in circular shape object

\subsubsection{Edge Detection}

Edge detection is used to detect points in an image which experience a sharp change in brightness. The lines can be obtained for feature detection and feature extraction. In this research, a circular landmark shape can be detected by using edge detection. Prewitt, Canny, Sobel are types of mathematical method have been developed for edge detection .Each of these methods uses a different approach in obtaining lines in an image, but can be grouped into two categories, gradient and Laplacian. The gradient method detects the edges by looking for the maximum and minimum in the first derivative of the image. The Laplacian method searches for zero crossings in the second derivative of the image to find edges.

The edge information in an image tends to be robust under changes in illumination or related camera parameters. Complex color-edge detection techniques exploit color changes between neighbouring pixels. Isotropic edge detectors that common used consider only the horizontal or vertical edge patterns to identifying the geometric structures of an image.

\subsubsection{Filtering}

Filtering is a nonlinear operation often used in image processing to reduce "salt and pepper' noise. Median filtering is more effective rather than convolution as it can reduce noise and obtain a sharp edge at the same times. The gray level of each pixel is replaced by the median of the gray levels in a neighborhood of that pixel. When median filtering is performed, each output pixel contains the median value in the $\mathrm{n} x \mathrm{n}$ neighborhood replace the corresponding pixel in the input image matrix. $3 \times 3$ neighborhood is common filter used as is the smallest dimension, utilizes less processing resources and thus saving time.

\subsubsection{Computation area pixel of image}

Pixel in image will be represented in two dimensional which are in $\mathrm{x}$ and $\mathrm{y}$ position. However, the array pixel has only one dimension, storing color values in linear sequence. In Matlab the regionprops command computes diferent properties of the image regions, such as centroid, area, perimeter, and bounding box. This command estimates the area of the objects in binary image. The result is represented in a scalar whose value corresponds roughly to the total number of on pixels in the image. Figure 9 show how a pixels stored in one-dimensional array. 


\section{How the pixels look:}

\begin{tabular}{|c|c|c|c|c|}
\hline 0 & 1 & 2 & 3 & 4 \\
\hline 5 & 6 & 7 & 8 & 9 \\
\hline 10 & 11 & 12 & 13 & 14 \\
\hline 15 & 16 & 17 & 18 & 19 \\
\hline 20 & 21 & 22 & 23 & 24 \\
\hline
\end{tabular}

\section{How the pixels are stored:}

\begin{tabular}{|l|l|l|l|l|l|l|l|l|l|l|l|l|l|l|}
\hline 0 & 1 & 2 & 3 & 4 & 5 & 6 & 7 & 8 & 9 & $\cdot$ & $\cdot$ & $\cdot$ & & \\
\hline
\end{tabular}

Figure 9. Pixels stored in one-dimensional array

\section{Results}

\subsection{Image Processing Method}

This experiment was conducted at the indoor environment. A set of capture image frames performed circular object recognition algorithm for VTOL was conducted in the indoor environment. VTOL can detecting the circular shape is maximum distance considered 50 centimeter (from the ground floor) and minimum distance is 20 centimeter (from the ground floor). This distance range is measured on starting from the ground floor and aligned to recognized object perpendicularly show in Figure 10. There are seven marks fixed at a distance of $20 \mathrm{~cm}, 25 \mathrm{~cm}, 30 \mathrm{~cm}, 35 \mathrm{~cm}, 40 \mathrm{~cm}, 45 \mathrm{~cm}$ and $50 \mathrm{~cm}$ from the ground floor. The VTOL can start from any point beyond $50 \mathrm{~cm}$ mark. A Graphical User Interface (GUI) is developed that consists of functions for VTOL such as show the result of tracking and position estimation of a circular object. The examples of GUI are show in Figure 10 and Figure 11.

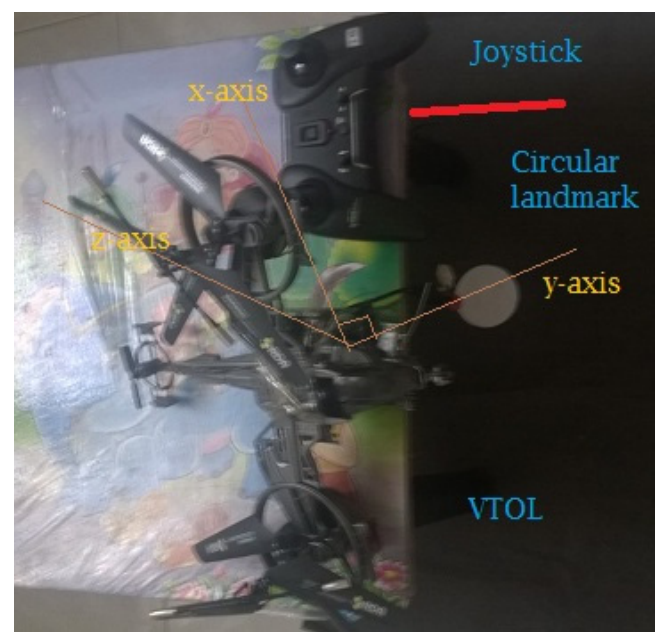

Figure 10. VTOL aligned to recognized landmark perpendicularly

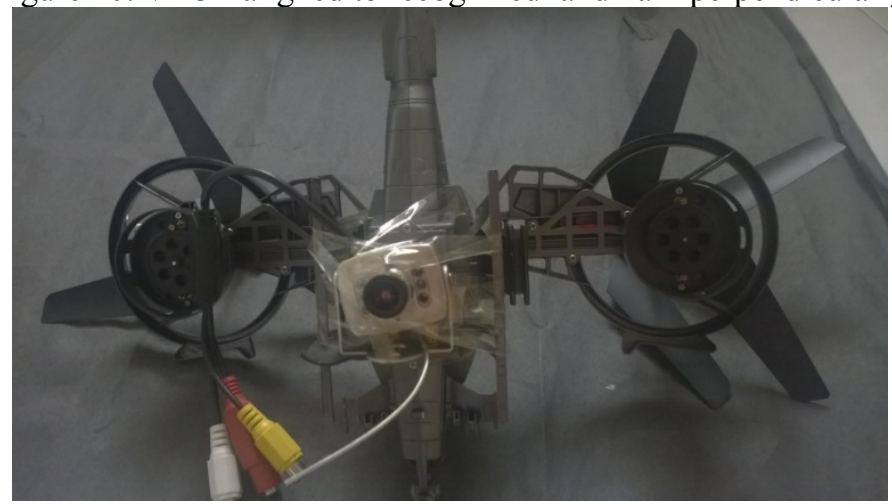

Figure 11. Camera mounted on VTOL facing downward to ground 


\subsection{Analysis of Testing Programming}

The position of centroid (x,y), area of circle in pixel and the estimated distance is compute and will be shown in the box show in Figure 12. Table 1 shows the result of area size in pixels is obtained form different height of camera refer to landmark. There are three sample of selected image which are capture at $20 \mathrm{~cm}, 25 \mathrm{~cm}, 30 \mathrm{~cm}$, $35 \mathrm{~cm}, 40 \mathrm{~cm}$ and $50 \mathrm{~cm}$. Then the average area size of circle will be calculated. The relationship between the height $(\mathrm{cm})$ of VTOL versus area size of circle is show in Figure 13. As seen in Figure 13, a mathematical models is obtained based on the height of camera for pose estimation algorithm.

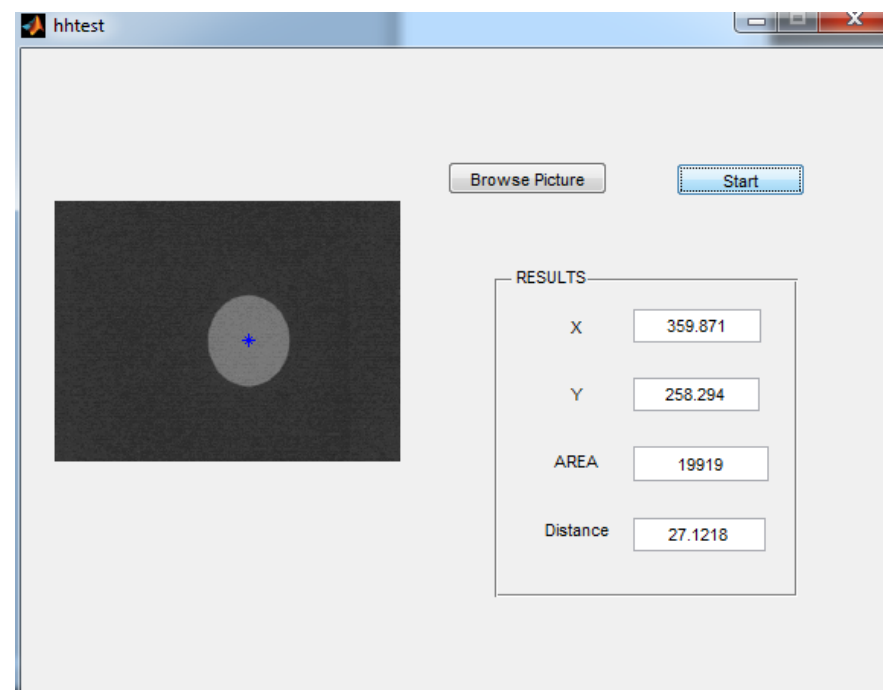

Figure 12. GUI interface

Table 1. Result of area size in pixels

\begin{tabular}{|c|c|c|c|c|}
\hline HEIGHT & AREA SIZE & IRCLE & & \\
\hline & 1 & 2 & 3 & Average \\
\hline 20 & 39566 & 39400 & 39741 & 39569 \\
\hline 25 & 26544 & 26512 & 25910 & 26322 \\
\hline 30 & 20262 & 19506 & 19500 & 19756 \\
\hline 35 & 15977 & 16013 & 16388 & 16126 \\
\hline 40 & 12486 & 11751 & 12822 & 12353 \\
\hline 50 & 6988 & 6813 & 8345 & 7382 \\
\hline
\end{tabular}

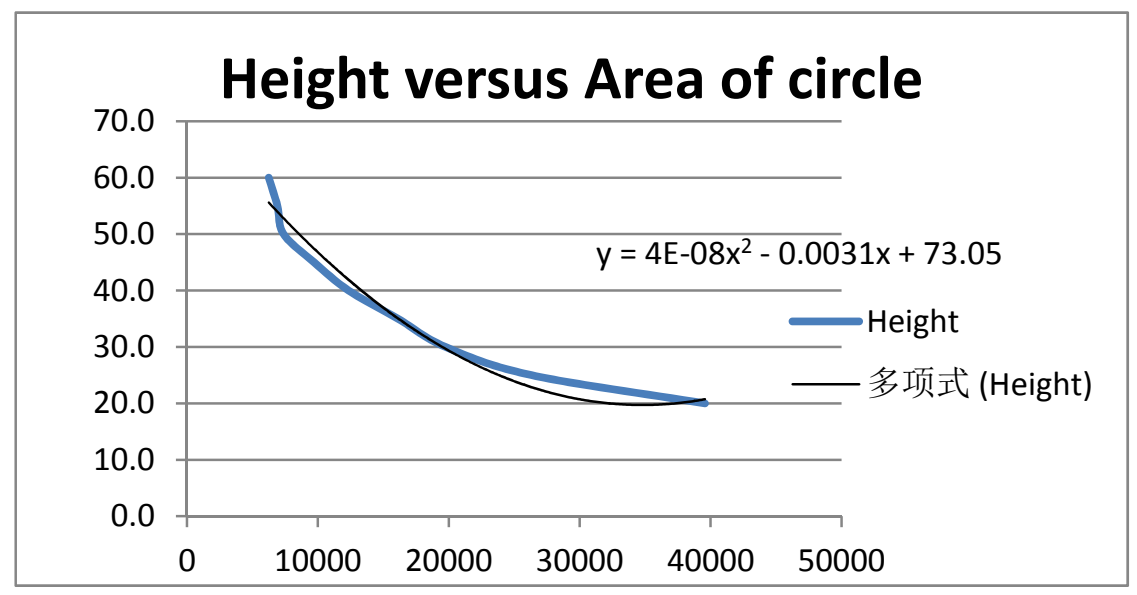

Figure 13. Relationship between height $(\mathrm{cm})$ and area size (pixel)

The result in Table 2 show the accuracy of desired height when comparing the actual height of VTOL with the calculated height obtained from the mathematical models in Figure 13. As seen in Table 2, the accuracy of desire 
height is almost $80 \%$ which is acceptable for actual height.

Table 2. Accuracy of desire height

\begin{tabular}{cccccccc}
\hline The desired height & \multicolumn{7}{c}{ Calculated height } \\
\hline & & Accuracy & Test & Accuracy & Test & Accuracy & accuracy \\
$(\mathrm{cm})$ & Test1 & $(\%)$ & 2 & $(\%)$ & 3 & $(\%)$ & $(\%)$ \\
20 & 16.3 & 81.5 & 15.8 & 79.0 & 15.4 & 77.0 & 79.2 \\
25 & 19.8 & 79.0 & 19.5 & 78.0 & 21.4 & 85.6 & 80.9 \\
30 & 26.5 & 88.3 & 24.3 & 81.0 & 25.5 & 85.0 & 84.78 \\
35 & 30.2 & 86.3 & 31.8 & 90.9 & 32.2 & 92.0 & 89.7 \\
40 & 36.0 & 90.0 & 37.4 & 93.5 & 37.0 & 92.5 & 92.0 \\
45 & 39.7 & 88.2 & 40.3 & 89.6 & 44.3 & 98.4 & 92.1 \\
50 & 46.0 & 92.0 & 49.4 & 98.8 & 47.4 & 94.8 & 95.2 \\
\hline
\end{tabular}

\section{Conclusion}

As conclusion, a vision based Vertical Take-off and Landing (VTOL) using one single landmark will be implemented with user interface system (GUI). The user can monitor the landmark recognition and navigate VTOL back to landmark process at anytime. The success of VTOL estimation position will depend on the accuracy of circular area size measurement. A more powerful sensors using in applications could influence the performance considerably. A high accuracy sensor such as stereo camera system has the potential to yield a higher level of reliability. These sensors will be able to obtain more accurate image features include improving range, sensors sensitivity, and incorporating complex computational capabilities.

\section{Acknowledgments}

The authors are grateful for the support granted by by Center for Robotics and Industrial Automation, Universiti Teknikal Malaysia Melaka (UTeM) in conducting this research through grant JURNAL/2018/FKE/Q00007 and Ministry of Higher Education.

\section{References}

Arvacheh, E.M. \& Tizhoosh, H.R. (2006). IRIS Segmentation: Detecting Pupil, Limbus and Eyelids, IEEE International Conference.https://doi.org/10.1109/ICIP.2006.312773

D. Eberli, D.Scaramuzza, S.Weiss \& R. Siegwar, (2010). Vision Based Position Control for MAVs Using One Single Circular Landmark, Springer, Switzerland.https://doi.org/10.1007/978-94-007-1110-5_29

Gohring, D. \& Burkhard, H.-D. (2006). Multi Robot Object Tracking and Self Localization Using Visual Percept Relations, Intelligent Robots and Systems, IEEE/RSJ International Conference, 31-36.https://doi.org/10.1109/IROS.2006.282427

G. Jang, S. Kim, W. Lee \& I. Kweon. (2003). Robust self-localization of mobile robots using artificial and natural landmarks. In IEEE Int. Symposium on Computational Intelligence in Robotics and Automation, 412-417.https://doi.org/10.1109/CIRA.2003.1222125

Haibo Huang, Dong Sun, Wuwei Chen \& Mills, J.K. (2005). A vision-based position control methodology to drive mobile robots towards target positions, IEEE International Conference on Robotics and Biomimetics (ROBIO), 369-374.https://doi.org/10.1109/ROBIO.2005.246295

Jae-Moon, Chung. \& Ohnishi, N. (2008). Cue circles: image feature for measuring 3-D motion of articulated objects using sequential image pair, Automatic Face and Gesture Recognition.

Lu fang, Gao., Yu-Xian, Gai. \& Sheng, Fu. (2007). Simultaneous Localization and Mapping for Autonomous Mobile Robots Using Binocular Stereo Vision System. International Conference on Mechatronics and Automation, 326-330.

Lu Wang, You. \& S. Neumann, U. (2007). Single View Camera Calibration for Augmented Virtual Environments. Virtual Reality Conference, 255-258.https://doi.org/10.1109/VR.2007.352494

Myong Ho Kim, Sang Cheol Lee \& Kwae-Hi Lee. (2001). Self-localization of mobile robot with single camera in corridor environment., 2001. IEEE International Symposium on Industrial Electronics, 1619-1623.

Pifu Zhang, Milios, E.E. \& Gu, J. (2004). Underwater Robot Localization using Artificial Visual 
Landmarks, Robotics and Biomimetics. IEEE International Conference on ROBIO, 705-710.

Qi Xiaoping, Zhang Qiheng, Ouyang Yimin \& Ma Jiaguang. (2006). A Method for Object Tracking using Shape Matching. IEEE Workshop on Signal Processing Systems Design and Implementation, 372-376.https://doi.org/10.1109/SIPS.2006.352611

Ray N., Acton S. T. \& Ley K. F. (2005). I Tracking leukocytes in vivo with shape and size constrained active $\begin{array}{lllll}\text { contours, } & \text { EEE Transactions } & \text { on }\end{array}$ 1222-1235.https://doi.org/10.1109/TMI.2002.806291

Thevenaz, P. \& Unser, M. (2006). The Snakuscule, International Conference on Image Processing, 1-12.

Won Kim \& Ju-Jang Lee. (2001). Visual tracking using Snake based on target's contour. Proceedings of Information Industrial, 1-12.https://doi.org/10.1109/ICIP.2006.312658

Yanmin Lao, Minghong Liao \& Zhipeeng Zhan. (2010). A Similarity Anlysis And Clustering Algorithm for Video Based on Moving Trajectory Time Series Wavelet Transform Of Moving Object In Video, International Conference Image Analysis and Signal Processing.https://doi.org/10.1109/IASP.2010.5476192

Yuen, D.C.K. \& MacDonald, B.A. (2005). Vision-based localization algorithm based on landmark matching, triangulation, reconstruction, and comparison, IEEE Transactions on Robotics, 21(2), 217-226.https://doi.org/10.1109/TRO.2004.835452

\section{Copyrights}

Copyright for this article is retained by the author(s), with first publication rights granted to the journal.

This is an open-access article distributed under the terms and conditions of the Creative Commons Attribution license (http://creativecommons.org/licenses/by/4.0/). 